\title{
A BOUNDARY LAYER RESULT FOR AN $n$-DIMENSIONAL LINEAR ELLIPTIC EQUATION
}

\author{
V. J. MIZEL ${ }^{1}$
}

Introduction. Since the publication of Levinson's results concerning the first boundary value problem for $\epsilon \Delta u+A(x, y) u_{x}+B(x, y) u y$ $+C(x, y) u=D(x, y)[6]$, there have been a number of other papers dealing with related two-dimensional problems $[1 ; 3 ; 7]$. More recently a paper by O. A. Ladyjzenskaya has appeared in which an $n$-dimensional extension of the original problem is discussed [4]. ${ }^{2}$ It is felt that the following treatment of the $n$-dimensional problem making use of a previously devised modification of Levinson's approach $[1 ; 3]$, may be of interest. It is readily seen that this treatment can be modified so as to include the case of certain types of singularities in the field of characteristics of the $\epsilon=0$ equation [7].

Roughly speaking, our object is to prove that the solution $u(x, \epsilon)$ of a certain $\epsilon$-parametered elliptic equation (1.1) may be characterized as the superposition of two functions. One of the two is a function $U(x)$ independent of $\epsilon$, while the other function $z(x, \epsilon)$ is of significant magnitude only near a boundary of the domain. That is, $z(x, \epsilon)$ is a "boundary layer" term.

The plan of the discussion is as follows. The hypotheses and the geometrical aspects of the problem are discussed in $\$ 1$. In $\$ 2$ the formal statement of the theorem is given and boundary layer terms are constructed. Finally, in $\$ 3$ the desired conclusion is proved by appeal to a maximum principle.

1. Consider the following Dirichlet problem:

$$
L_{\epsilon} u=\epsilon a_{i j}(x) u_{, i j}+a_{i}(x) u_{, i}+c(x) u=d(x) \quad u=\tilde{u}(x) \text { on } D .
$$

Here $L_{\epsilon}$ is a uniformly elliptic operator obeying a maximum principle $\left(u_{, i}\right.$ denotes $\partial u / \partial x_{i}$ and analogously for $\left.u_{, i j}\right), x=\left(x_{1}, x_{2}, \cdots, x_{n}\right)$ denotes a point of $E^{n}$, the summation convention is utilized, and $D$ is a bounded domain of $E^{n}$ with its boundary denoted by $\dot{D}$.

More precisely, the hypotheses are:

(1) The domain $D$ is of class $C_{[n / 2]+3}$ in the sense that it contains

Presented to the Society, October 25, 1957; received by the editors November 25, 1958.

1 The author is now associated with Carnegie Institute of Technology.

2 The author wishes to express his appreciation to Dr. D. G. Aronson for calling this paper to his attention. 
subdomains $A_{1}, A_{2}, \cdots, A_{m}$, covering all of $D$ except for a compact subset, whose closures are class $C_{[n / 2]+3}$ homeomorphs of closed bounded domains in $E^{n}$. The images of the sets $\bar{A}_{k} \cap \dot{D}$ under these homeomorphisms, whose inverses also are of class $C_{[n / 2]+3}$, are $(n-1)$ dimensional planar subsets of $E^{n}$.

(2) The symmetric matrix $a_{i j}(x)$ is uniformly positive definite on $\bar{D}$, so that whenever $x \in \bar{D} \quad \xi^{i} a_{i j}(x) \xi^{i} \geqq \alpha \xi^{i} \xi^{i}$ for every vector $\xi$ $=\left(\xi^{1}, \cdots, \xi^{n}\right)$ and a fixed constant $\alpha>0$.

(3) $c(x)$ is negative throughout $\bar{D}$. $\bar{D}$.

(4) $a_{i j}(x) \in C_{[n / 2]+2}, \tilde{u}(x) \in C_{[n / 2]+3}$ and $a_{i}(x), c(x), f(x) \in C_{[n / 2]+1}$ on

Inasmuch as the following development does not require functions to be smoother than $C_{4}$ except to ensure existence of $u(x, \epsilon),(1)$ and (4) may be replaced by such weaker conditions as achieve the same end $[8]$.

We now define a "tube." Let $S^{p} p=1,2$ denote closed sets contained in disjoint open subsets $\oint^{p}$ of $\dot{D}$, where we suppose the following conditions to be satisfied:

(I) $\mathcal{S}^{p}, p=1,2$, is the homeomorph, by a $C_{4}$ mapping $J^{p}$, of a domain in the hyperplane $\nu_{n}=0$. [For clarity we denote points of $E^{n}$ by $\nu=\left(\nu_{1}, \nu_{2}, \cdots, \nu_{n}\right)$ when discussing homeomorphs of subsets of $\left.\dot{D}_{\text {.. }}\right]$

(II) On $S^{p}$ the vector field $a_{i}(x)$ is nowhere orthogonal to the field $n_{i}(x)$ of into- $D$-directed unit normal vectors to $\dot{D}$; that is, $a_{i}(x)$ is nowhere tangent to $S^{p}$. Moreover all solutions of $d x_{1} / a_{1}(x)=d x_{2} / a_{2}(x)$ $=\cdots=d x_{n} / a_{n}(x)$ which intersect $S^{1}$ intersect $\dot{D}$ next through $S^{2}$, and conversely. To be specific we denote by $S^{1}$ that one of the sets $S^{p}$ on which the characteristics of $L_{0} u$ (i.e. $L_{\epsilon} u$ with $\epsilon=0$ ), defined by:

$$
d x_{i} / d t=-a_{i}(x),
$$

enter $D$ with increasing $t$. Thus,

$$
a_{i}(x) n_{i}(x)<0 \text { on } S^{1}, \quad a_{i}(x) n_{i}(x)>0 \text { on } S^{2} .
$$

(III) $S^{1}$ is the homeomorphic image under $J^{1}$ of a convex closed domain $V^{1} \subset\left(J^{1}\right)^{-1} \delta^{1}$. The set $X$ traced out by those characteristics of $L_{0} u$ joining $S^{1}$ and $S^{2}$ is a "tube."

It follows from the above hypotheses that $S^{2}$ is also a homeomorph of $V^{1}$ and that $X$ is an $n$-dimensional set. These statements are readily verified. Note to begin with that $S^{2}$ may be characterized by:

$$
\begin{aligned}
J^{2}\left(\nu_{1}^{2}, \cdots, \nu_{n-1}^{2}, 0\right)=x^{2}=F(t, & \left.x_{1}^{1}, \cdots, x_{n}^{1}\right), \\
x^{p} & \in S^{p}, \nu^{2} \in\left(J^{2}\right)^{-1} S^{2} .
\end{aligned}
$$


Here $x=F\left(t, x^{1}\right)$ denotes the characteristic solving:

$$
\frac{d x_{i}}{d t}=-a_{i}(x), \quad x_{i}(0)=x_{i}^{1} .
$$

Rewriting (1.4) leads to:

$$
\begin{aligned}
J^{2}\left(\nu_{1}^{2}, \cdots, \nu_{n-1}^{2}, 0\right)-F\left(t, J_{1}^{1}(\nu), \cdots,\right. & \left.J_{n}^{1}\left(\nu^{1}\right)\right)=0, \\
& \nu^{1} \in V^{1}, \nu^{2} \in\left(J^{2}\right)^{-1} S^{2} .
\end{aligned}
$$

Now it follows from our hypotheses that there is associated with each $\nu^{1}$ a unique $\nu^{2}$ and $t$, i.e. the values corresponding to the (first) $S^{2}$ intersection of the characteristic passing through $x^{1}=J^{1}\left(\nu^{1}\right)$. The functions $t\left(\nu^{1}\right)$ and $\nu_{i}^{2}\left(\nu^{1}\right), i=1, \cdots, n-1$, obtained in this fashion are of class $C_{4}$. For the $n-1$ tangent vectors $J_{v_{i}}^{2_{2}}\left(\nu_{1}^{2}, \cdots, \nu_{n-1}^{2}, 0\right)$, $i=1, \cdots, n-1$, [henceforth a comma preceding a subscript denotes partial differentiation ] are certainly linearly independent on $S^{2}$, while the vector $F_{, t}\left(t, J_{1}^{1}\left(\nu^{1}\right), \cdots, J_{n}^{1}\left(\nu^{1}\right)\right)=-a\left(x^{2}\right)$ is not tangent to $S^{2}$, so that these $n$ vectors form a linearly independent set on $S^{2}$. Therefore the implicit function theorem may be applied to $\left(1.4_{1}\right)$ to prove the $C_{4}$ character of $t\left(\nu^{1}\right), \nu_{i}^{2}\left(\nu^{1}\right)$. Moreover, since the vectors $F_{, \nu_{i}^{1}}\left(t, J_{1}^{1}\left(\nu^{1}\right), \cdots, J_{n}^{1}\left(\nu^{1}\right)\right)$ and $F_{, t}\left(t, J_{1}^{1}\left(\nu^{1}\right), \cdots, J_{n}^{1}\left(\nu^{1}\right)\right)$ are all solutions of the linear differential system:

$$
\frac{d y_{i}}{d t}=-a_{i, j}\left[F\left(t, \Im_{1}^{1}\left(\nu^{1}\right), \cdots, \Im_{n}^{1}\left(\nu^{1}\right)\right)\right] y_{j}
$$

their obvious linear independence on $S^{1}$ ensures their linear independence throughout $X$ (see [2, pp. 25-26]). This demonstrates the $n$ dimensionality of $X$ and, in addition, shows that the implicit function theorem may be also applied to $\left(1.4_{1}\right)$ to prove the existence of unique $C_{4}$ functions $t\left(\nu^{2}\right), \nu_{j}^{1}\left(\nu^{2}\right) j=1, \cdots, n-1$. Note that this ensures that the mapping $\nu_{i}^{2}\left(\nu^{1}\right)$ is of rank $n-1$.

In concluding this section, we note that the hypotheses on the subsets $S^{p}$ are of such nature as to ensure equally well the existence of correspondingly related pairs $O^{p}, P^{p}, Q^{p}$ with $\AA^{p}>O^{p}>P^{p}>Q^{p}>S^{p}$ [">" denotes "is contained in the interior of"]. The new sets $O^{1}$, $P^{1}, Q^{1}$ may also be supposed homeomorphic under $J^{1}$ to convex compact domains in $\left(J^{1}\right)^{-1} \delta^{1}$. We shall denote the tubes generated in this way by $X_{o}, X_{P}, X_{Q}$, respectively.

2. We return now to:

$$
L_{\epsilon} u=\epsilon a_{i j}(x) u_{, i j}+a_{i}(x) u_{, i}+c(x) u=d(x) \quad u=\tilde{u}(x) \text { on } \dot{D} .
$$


The hypotheses introduced in $\S 1$ ensure that there exists in $\bar{D}$ a unique solution $u(x, \epsilon)$ for each $\epsilon>0$. We shall show that, for small $\epsilon$, $u(x, \epsilon)$ consists essentially of the superposition of the "boundary layer" term $z(x, \epsilon)$ with a solution $U(x)$ of the reduced equation:

$$
L_{0} U=a_{i}(x) U_{, i}+c(x) U=d(x) .
$$

More precisely, we shall prove the

THEOREM. In $X$ there occurs the following decomposition

$$
u(x, \epsilon)=U(x)+z(x, \epsilon)+w(x, \epsilon),
$$

where: $U(x)$ is that solution of the reduced equation coinciding with $\tilde{u}(x)$ on $S^{1} ; z(x, \epsilon)$ has in a neighborhood of $S^{2}$ the form $z=h(x) e^{-g(x) / \epsilon}$ (with $g=0$ on $S^{2}, g>0$ off of $S^{2}$ ), and elsewhere in $X$ is uniformly $O\left(e^{-\delta_{1} / \epsilon}\right)$ for a fixed positive $\delta_{1} ; w(x, \epsilon)$ is uniformly $O\left(\epsilon^{1 / 2}\right)$ in $X$ and vanishes on $S^{p}$.

The method of proof will be to obtain boundary layer quantities $h(x) e^{-g(x) / \epsilon}$ and $H(x) e^{-G(x) / \epsilon^{1 / 2}}$ which satisfy $L_{\epsilon} u=0$ uniformly to within $O(\epsilon)$ and $O\left(\epsilon^{1 / 2}\right)$, respectively. We extend the initial domain of definition of $z(x, \epsilon)=h(x) e^{-g(x) / \epsilon}$ by continuing this term in a $C_{2}$, uniformly $O\left(e^{-\delta_{1} / \epsilon}\right)$ fashion to all of $X_{Q}$. Then we utilize the maximum principle for $L_{\epsilon}$ to show that the difference between $u(x, \epsilon)$ and the superposition of $U(x), H(x) e^{-G(x) / \epsilon^{1 / 2}}$ and $z(x, \boldsymbol{\epsilon})$ is uniformly $O\left(\boldsymbol{\epsilon}^{1 / 2}\right)$ on $X_{Q}$. Since $H(x) e^{-G(x) / \epsilon^{1 / 2}}$ is itself uniformly $O\left(\epsilon^{1 / 2}\right)$ on $X$, the conclusion of the theorem results.

To begin with, substitution of the expressions $h(x) e^{-\sigma(x) / \epsilon}$ and $H(x) e^{-G(x) / \epsilon^{1 / 2}}$ into $L_{\epsilon} u=0$ reveals that they will each formally satisfy this equation to the required degree of approximation provided the following conditions are met:

$$
\begin{aligned}
a_{i j}(x) g_{, i} g_{, j}-a_{i}(x) g_{, i} & =0, \\
\left(a_{i}(x)-2 a_{i j}(x) g_{, j}\right) h_{, i}+\left(c(x)-a_{i j}(x) g_{, i j}\right) h & =0 \\
a_{i}(x) G_{, i} & =0 \\
a_{i}(x) H_{, i}+\left(c(x)+a_{i j}(x) G_{, i} G_{, j}\right) H & =0 .
\end{aligned}
$$

We now set the following initial value problem for the nonlinear $g$ equation in (2.3):

$$
a_{i j}(x) g_{, i} g_{, j}-a_{i}(x) g_{, i}=0 \quad g\left(x^{2}\right)=0, x^{2} \in O^{2},
$$

and we shall seek a solution other than the trivial one $g \equiv 0$. Writing the characteristic equations associated with $\left(2.3_{1}\right)$ we obtain: 


$$
\begin{aligned}
& \frac{d}{d \tau}\left(x_{k}\right)=2 a_{k j}(x) g_{, j}-a_{k}(x), \\
& \frac{d}{d \tau}\left(g_{, k}\right)=-\left(a_{i j}(x)\right)_{, k} g_{, i} g_{, j}-\left(a_{i}(x)\right)_{, k} g_{, i}, \\
& \frac{d}{d \tau}(g)=a_{i j}(x) g_{, i} g_{, j} .
\end{aligned}
$$

It is well known that if $C_{3}$ initial values chosen on $O^{2}$ for the $g_{, k}$ satisfy $\left(2.3_{1}\right)$, are consistent with the initial value for $g$, and determine $O^{2}$ as a noncharacteristic manifold, then there exists in a neighborhood of $O^{2}$ a unique $C_{4}$ solution to $\left(2.3_{1}\right)$ satisfying the initial conditions on both the $g, k$ and $g[5]$.

In order to show that initial values for $g, k$ may in addition be so chosen as to yield a nontrivial solution, we note that the given initial value for $g$ certainly requires:

$$
\begin{aligned}
g_{, k}\left(x^{2}\right)= & \lambda\left(x^{2}\right) n_{k}\left(x^{2}\right), \quad x^{2} \in O^{2}, \\
& \text { with } \lambda\left(x^{2}\right) \text { an as yet unspecified function. }
\end{aligned}
$$

Substitution into $\left(2.3_{1}\right)$ leads to:

$$
\left[\lambda\left(x^{2}\right)\right]^{2} a_{i j}\left(x^{2}\right) n_{i}\left(x^{2}\right) n_{j}\left(x^{2}\right)-\lambda\left(x^{2}\right) a_{i}\left(x^{2}\right) n_{i}\left(x^{2}\right)=0 .
$$

Therefore, since $a_{i j}\left(x^{2}\right) n_{i}\left(x^{2}\right) n_{j}\left(x^{2}\right) \geqq \alpha>0$ and $a_{i}\left(x^{2}\right) n_{i}\left(x^{2}\right)>0$, we can choose:

$$
\lambda\left(x^{2}\right)=\frac{a_{i}\left(x^{2}\right) n_{i}\left(x^{2}\right)}{a_{i j}\left(x^{2}\right) n_{i}\left(x^{2}\right) n_{j}\left(x^{2}\right)}>0 .
$$

Thus provided with obviously nontrivial $C_{3}$ initial values for the $g_{, k}$ we can verify that the remaining condition is satisfied. By $\left(2.3_{1}\right)$ and $\left(2.3_{2}\right)$ we find:

$$
\begin{aligned}
n_{k}\left(x^{2}\right) \frac{d}{d \tau}\left(x_{x}^{2}\right) & =\left[\lambda\left(x^{2}\right)\right]^{-1}\left[2 a_{k j}\left(x^{2}\right) g_{, k} g_{, j}-a_{k}\left(x^{2}\right) g_{, k}\right] \\
& =\left[\lambda\left(x^{2}\right)\right]^{-1} g_{, k} a_{k}\left(x^{2}\right)=n_{k}\left(x^{2}\right) a_{k}\left(x^{2}\right)>0,
\end{aligned}
$$

so that $O^{2}$ is a noncharacteristic manifold with the above choice for $g_{, k}\left(x^{2}\right)$. In particular, solutions of $\left(2.3_{2}\right)$ enter $D$ with increasing $\tau$. Further we see that $\left(2.3_{2}\right)$ leads to:

$$
\frac{d}{d \tau}\left(g\left(x^{2}\right)\right)=a_{i j}\left(x^{2}\right) g_{, i} g, j \geqq \alpha\left[\lambda\left(x^{2}\right)\right]^{2}>0,
$$


ensuring the existence of a positive solution $g(x)$ throughout a (deleted) neighborhood of $O^{2}$. Moreover (2.9) implies that for a sufficiently small constant $\tau_{1}>0$ there exists a $\bar{\delta}>0$ such that:

$$
g\left(\tau, x^{2}\right)>\bar{\delta} \tau \quad 0<\tau \leqq \tau_{1}, x^{2} \in O^{2} .
$$

Now we introduce a $C_{3}$ function $\phi\left(x^{2}\right)$ defined on $O^{2}$ and positive except on $\dot{O}^{2}$. For a sufficiently small constant $\gamma>0$ we see that $\gamma \phi\left(x^{2}\right) \leqq \tau_{1}$ so that the set:

$$
\omega_{1}: x=J\left(\tau, x^{2}\right) \quad 0<\tau \leqq \gamma \phi\left(x^{2}\right), x^{2} \in O^{2}
$$

is a subset of the set characterized by (2.10). Here $x=J\left(\tau, x^{2}\right)$ denotes the base characteristic of $\left(2.3_{1}\right)$ passing through $x^{2}$, i.e. a solution of the first system of $\left(2.3_{2}\right)$.

Moreover the constant $\gamma$ may be chosen sufficiently small so that the surface

$$
W_{1}: x=J\left(\gamma \phi\left(x^{2}\right), x^{2}\right)
$$$$
x \in O^{2}
$$

is "almost parallel" to $O^{2}$ in the sense that there exists a constant $\sigma_{1}>0$ for which the following is a subset of $D$ :

$$
\omega_{2}: x=x^{w}+\sigma N\left(x^{w}\right) \quad x^{w} \in W_{1}, 0 \leqq \sigma \leqq \sigma_{1} .
$$

$N\left(x^{w}\right)$ here denotes the field of out-of- $\omega_{1}$ directed unit normal vectors to $W_{1}$. Such almost parallelism occurs when $N_{i}\left(x^{w}\right) n_{i}\left(x^{2}\right)$ is positive for all $x^{w}=x^{2} \in \dot{O}^{2}$.

That $W_{1}$ is indeed an $n-1$ surface and that $\omega_{1}$ is $n$-dimensional follows from the linear dependence in $O^{2}$ of the $n$ vectors

$$
\begin{aligned}
&(2.14) J_{, \tau}\left(\tau, \mathrm{J}_{1}^{2}\left(\nu^{2}\right), \cdots, \mathrm{J}_{n}^{2}\left(\nu^{2}\right)\right), \quad J_{, \nu_{i}}^{2}\left(\tau, \mathrm{J}_{1}^{2}\left(\nu^{2}\right), \cdots, \mathrm{T}_{n}^{2}\left(\nu{ }^{2}\right)\right) \\
& i=1, \cdots, n-1,
\end{aligned}
$$

all of which are solutions of a certain $C_{2}$ linear system of ordinary differential equations [2]. For these vectors are thereby linearly independent and of class $C_{2}$ throughout $\omega_{1}$, and the remaining details are strictly analogous to those discussed in $\$ 1$. Furthermore the knowledge that, for small $\gamma, W_{1}$ is almost parallel to $O^{2}$ follows from the same linear independence and smoothness, for the $n-1$ tangent vectors to $W_{1}, J_{, \nu_{i}^{2}}\left[\gamma \phi\left(x^{2}\left(\nu^{2}\right)\right), J_{1}^{2}\left(\nu^{2}\right), \cdots, J_{n}^{2}\left(\nu^{2}\right)\right], i=1, \cdots, n-1$, are then almost equal to the corresponding tangent vectors to $O^{2}$ $J_{, \nu_{i}^{2}}\left(0, J_{1}^{2}\left(\nu^{2}\right), \cdots, J_{n}^{2}\left(\nu^{2}\right)\right)$ and are also linearly independent.

We note further that the linear independence of the $n$ vectors $\left[x^{w}\left(\nu^{2}\right)+\sigma N\left(x^{w}\left(\nu^{2}\right)\right)\right]_{, \nu_{i}^{2}}$ and $\left[x^{w}\left(\nu^{2}\right)+\sigma N\left(x^{w}\left(\nu^{2}\right)\right)\right]_{, \sigma}$ on $W_{1}$ ensures their linear independence in a neighborhood of $W_{1}$. That $\sigma_{1}$ can be 
chosen sufficiently small so that there is a one-one $C_{2}$ correspondence in $\omega_{2}$ between points $x$ and pairs $\left(\sigma, x^{w}\left(\nu^{2}\right)\right)$ then follows from the implicit function theorem.

Turning now to the second equation of (2.3), the linear equation involving $h$, we note that its base characteristics coincide with those previously obtained for the $g$ equation. Therefore we are assured that the following linear initial value problem has a unique $C_{2}$ solution throughout $\omega_{1}$ :

$$
\begin{array}{r}
\left(a_{i}(x)-2 a_{i j}(x) g_{, j}\right) h_{, i}+\left(c(x)-a_{i j}(x) g_{, i j}\right) h=0, \\
h=\left(\tilde{u}\left(x^{2}\right)-U\left(x^{2}\right)\right) \psi\left(x^{2}\right) \text { on } O^{2},
\end{array}
$$

where $\psi\left(x^{2}\right)$ denotes a $C_{2}$ function defined on $O^{2}$ which is identically unity on $Q^{2}$ and vanishes outside $P^{2}$, and $U(x)$ denotes that solution of the reduced equation described in the theorem. That $U(x)$ is uniquely defined and of class $C_{2}$ throughout $X_{P}$ follows from the hypothesized nontangency to $S^{P}$ of the base characteristics of (2.1) and smoothness hypothesis (4). It follows from well known existence theory of ordinary differential equations that the set $M_{1}$ consisting of all points $x=J\left(\gamma \phi\left(x^{2}\right), x^{2}\right)$ with $x^{2} \in P^{2}$ is a compact subset contained in the interior of $W_{1}$. In view of the homogeneity of $\left(2.3_{3}\right)$ it is clear that $h$ vanishes together with its partial derivatives at all points of $W_{1}$ not lying in $M_{1}$.

We now deal directly with the product $z(x, \epsilon)=h(x) e^{-g(x) / \epsilon}$. This function is defined in $\omega_{1}$ and vanishes together with its partial derivatives at all points of $W_{1}-M_{1}$. Denote by $z_{, N}\left(x^{w}, \epsilon\right)$ and $z_{, N N}\left(x^{w}, \epsilon\right)$ the first and second order normal derivatives of $h(x) e^{-g(x) / \epsilon}$ on $W_{1}$. We extend $z(x, \epsilon)$ throughout $\omega_{2}$ as follows:

$$
z(x, \epsilon)=\left[A\left(x^{w}\right)+\sigma B\left(x^{w}\right)+\sigma^{2} C\left(x^{w}\right)\right]\left(1-\frac{\sigma}{\sigma_{1}}\right)^{3} 0 \leqq \sigma \leqq \sigma_{1},
$$

where

$$
\begin{aligned}
A\left(x^{w}\right) & =z\left(x^{w}, \epsilon\right) \\
B\left(x^{w}\right) & =z, N\left(x^{w}, \epsilon\right)+\frac{3}{\sigma_{1}} A\left(x^{w}\right)=z_{, N}\left(x^{w}, \epsilon\right)+\frac{3}{\sigma_{1}} z\left(x^{w}, \epsilon\right), \\
C\left(x^{w}\right) & =\frac{1}{2} z, N N\left(x^{w}, \epsilon\right)+\frac{3}{\sigma_{1}} B\left(x^{w}\right)-\frac{3}{\left(\sigma_{1}\right)^{2}} A\left(x^{w}\right) \\
& =\frac{1}{2} z_{, N N}\left(x^{w}, \epsilon\right)+\frac{3}{\sigma_{1}} z_{, N}\left(x^{w}, \epsilon\right)+\frac{6}{\left(\sigma_{1}\right)^{2}} z\left(x^{w}, \epsilon\right) .
\end{aligned}
$$


That (2.15) defines $z(x, \epsilon)$ as a bona fide function of $x$ is assured by the one-one relation between $\left(\sigma, x^{w}\left(\nu^{2}\right)\right)$ and $x$ in $\omega_{2}$. Moreover $z(x, \epsilon)$ as thus defined is seen to vanish together with its first and second order partial derivatives at all boundary points of $\omega_{1} \cup \omega_{2}$ excepting points of $P^{2}$. Hence we extend $z(x, \epsilon)$ in a $C_{2}$ manner to all of $X_{Q}$ by simply specifying the values in $X{ }_{Q} \cap\left(\omega_{1} \cup \omega_{2}\right)$ to be as given above and defining $z(x, \epsilon)$ to be identically zero elsewhere in $X_{Q}$. Note further that (2.10) ensures that $g\left(x^{w}\right)>2 \delta_{1}$ on $M_{1}$ for some constant $\delta_{1}>0$ since $\gamma \phi\left(x^{2}\right)$ is positive for $x^{2} \in P^{2}$. Therefore since $z\left(x^{w}, \epsilon\right), z, N\left(x^{w}, \epsilon\right)$ and $z_{, N N}\left(x^{w}, \epsilon\right)$ all include the factor $e^{-g\left(x^{w}\right) / \epsilon}$ it is evident that $z(x, \epsilon)$ as defined by (2.15), together with its partial derivatives of first and second order, is uniformly $O\left(e^{-\delta_{1} / \epsilon}\right)$ throughout $\omega_{2}$. The extension of $z(x, \epsilon)$ to all of $X$ in the manner described by the theorem has obviously been accomplished.

In order to obtain the term $H(x) e^{-G(x) / \text { el/2 }^{1 / 2}}$ we note that the first equation of (2.4) requires simply that $G(x)$ be constant along base characteristics of the reduced equation. We may therefore define $G(x)$ as a $C_{3}$ function in $X_{Q}$ by the following equation:

$$
G\left[F\left(t, J^{1}\left(\nu^{1}\right)\right)\right]=\kappa f\left(\nu^{1}\right) \quad \nu^{1} \in\left(J^{1}\right)^{-1} Q^{1}
$$

where $f\left(\nu^{1}\right)$ denotes a $C_{3}$ non-negative function vanishing only for $\nu^{1} \in\left(J^{1}\right)^{-1} \dot{Q}^{1}$, and where $\kappa$ is a positive constant to be specified below. That (2.16) unambiguously defines $G(x)$ as a $C_{3}$ function in $X_{Q}$ is a direct consequence of the previously discussed properties of the mappings $J^{1}\left(\nu^{1}\right)$ and $F\left(t, x^{1}\right)$.

Turning now to the equation in (2.4) for $H(x)$ we note that its base characteristics also coincide with those of the reduced equation. Moreover if $\kappa$ is chosen sufficiently small it is clear from our definition of $G(x)$ that $H(x)$ steadily increases along the characteristics as they proceed from $Q^{2}$ to $Q^{1}$. We are assured that the following linear initial value problem has a unique $C_{2}$ solution throughout $X_{Q}$ :

$$
\begin{aligned}
& a_{i}(x) H_{, i}+\left(c(x)+a_{i j}(x) G_{, i} G_{, j}\right) H=0, \\
& H\left(x^{2}\right) \equiv 2 M+K+1 \text { on } Q^{2} .
\end{aligned}
$$

Here $M$ denotes a bound for $|u(x, \epsilon)|$ on $\bar{D}$ as furnished by the maximum principle [e.g. $\left.M=\left(\inf _{\bar{D}}|c(x)|\right)^{-1} \sup _{\bar{D}}|d(x)|+\sup _{\dot{D}}|\tilde{u}(x)|\right]$ and $K$ denotes a bound for $|U(x)|$ on $X_{Q}$.

In the light of our construction it is evident that $H(x) e^{-G(x) / \epsilon^{1 / 2}}$ $\geqq 2 M+K+1$ on those characteristics in $X_{Q}$ passing through $\dot{Q}^{1}$ (and therefore passing through $\dot{Q}^{2}$, and that $H(x) e^{-G(x) / \epsilon^{1 / 2}}>0$ throughout $X_{Q}$. 
3. Consider now the $C_{2}$ functions

$$
\begin{aligned}
& \alpha(x, \epsilon)=U(x)+z(x, \epsilon)+H(x) e^{-G(x) / \epsilon^{1 / 2},} \\
& \beta(x, \epsilon)=U(x)+z(x, \epsilon)-H(x) e^{-G(x) / \epsilon^{1 / 2}}
\end{aligned}
$$

which are defined for $x \in X_{Q}$. By virtue of equations (2.3), (2.4) it is easily seen that $\left|L_{\epsilon} \alpha-d(x)\right|$ and $\left|L_{\epsilon} \beta-d(x)\right|$ are both uniformly $O\left(\epsilon^{1 / 2}\right)$. Moreover we note that the following inequalities hold for small $\epsilon$ :

$$
\alpha(x, \epsilon)>u(x, \epsilon)>\beta(x, \epsilon) \quad \text { when } x \in \dot{X}_{Q},
$$

where $\dot{X}_{Q}$ consists of $Q^{1} \cup Q^{2}$ together with those points traced out by the characteristics in $X_{Q}$ passing through $\dot{Q}^{1}$. Since $\alpha(x, \epsilon)-u(x, \epsilon)$ satisfies $L_{\epsilon} u=0$ to $O\left(\epsilon^{1 / 2}\right)$ and is non-negative on the boundary of $X_{Q}$ it follows that any negative minimum of this function is uniformly $O\left(\epsilon^{1 / 2}\right)$, so that $u(x, \epsilon)<\alpha(x, \epsilon)+O\left(\epsilon^{1 / 2}\right)$ certainly holds throughout $X_{Q}$. A similar argument shows that $u(x, \epsilon)>\beta(x, \epsilon)-O\left(\epsilon^{1 / 2}\right)$ throughout $X_{Q}$. Hence $\beta(x, \epsilon)-O\left(\epsilon^{1 / 2}\right)<u(x, \epsilon)<\alpha(x, \epsilon)+O\left(\epsilon^{1 / 2}\right)$ throughout $X_{Q}$. Therefore we may write:

$$
\begin{aligned}
|u(x, \epsilon)-(U(x)+z(x, \epsilon))|<H(x) e^{-G(x) / \epsilon^{1 / 2}}+ & O\left(\epsilon^{1 / 2}\right) \\
& \text { when } x \in X_{Q} .
\end{aligned}
$$

Inasmuch as $H(x) e^{-G(x) / \epsilon^{1 / 2}}$ is itself uniformly $O\left(\epsilon^{1 / 2}\right)$ on $X$, we have proved the theorem stated in $\$ 2$.

\section{BIBLIOGRAPHY}

1. D. G. Aronson, Linear parabolic differential equations containing a small parameter, J. Rational Mech. Anal. vol. 5 (1956) pp. 1003-1014.

2. E. Coddington and N. Levinson, Theory of ordinary differential equations, McGraw-Hill, 1955.

3. S. L. Kamenomostskaya, On equations of elliptic and parabolic type with a small parameter in the highest derivative, Mat. Sb. vol. 31 (1952) pp. 703-708.

4. O. A. Ladyjzenskaya, On the equations with small parameter at the highest derivatives in the linear partial differential equations, Vestnik Leningrad. Univ. no. 7 (1957) pp. 104-120.

5. P. D. Lax, Partial differential equations, Notes, New York University, Institute of Mathematical Sciences, 1950.

6. N. Levinson, The first boundary value problem for $\epsilon \Delta u+A(x, y) u_{x}+B(x, y) u_{y}$ $+C(x, y) u=D(x, y)$ for small $\epsilon$, Ann. of Math. vol. 51(1950) pp. 428-445.

7. V. J. Mizel, $A$ boundary layer problem for an elliptic equation in the neighborhood of a singular point, Proc. Amer. Math. Soc. vol. 8 (1957) pp. 62-67.

8. L. Nirenberg, Remarks on strongly elliptic partial differential equations, Comm. Pure Appl. Math. vol. 8 (1955) pp. 648-674.

UNIVERSITY OF TENNESSEe 